\title{
Yb:CALGO bulk oscillator generating ultrashort pulses at high efficiency by cross-polarized optical pumping
}

\author{
François Labaye ${ }^{1}$, Valentin J. Wittwer ${ }^{1}$, Norbert Modsching ${ }^{1}$, Olga Razskazovskaya $^{1}$, Eric Cormier ${ }^{2,3}$, \\ Thomas Südmeyer ${ }^{1}$
}

1. Laboratoire Temps-Fréquence, Institut de Physique, Université de Neuchâtel, Avenue de Bellevaux 51, 2000 Neuchâtel, Switzerland

2. Laboratoire Photonique, Numérique et Nanosciences, UMR 5298, CNRS-IOGS-Université Bordeaux, 33400 Talence, France

3. Institut Universitaire de France (IUF), 1 rue Descartes, 75231 Paris

Numerous applications rely on transform-limited few-cycle laser pulses and are typically based on green-pumped Ti:sapphire laser oscillators [1,2]. Simple and cost-efficient Yb-doped bulk laser oscillators are a promising alternative, but have so far been severely limited in power and efficiency for operation in the sub-30-fs regime $[3,4]$. Standard colinearly-pumped Yb-doped bulk laser oscillators use dichroic pump mirrors with a high transmission for the pump at around $980 \mathrm{~nm}$ and a high reflectivity at the lasing wavelengths [Fig. 1a)]. This leads to a strongly varying group delay dispersion (GDD) and limits the expansion of the optical spectrum towards short wavelengths [5]. Furthermore, to reach higher power levels, higher output coupling is required, resulting in a stronger population inversion. For common broadband Yb-doped gain materials, this shifts the gain maximum towards shorter wavelengths until reaching the spectral edge of the dichroic mirrors. Both reasons set a severe limitation to fully exploit the available gain bandwidth for the gereation of even shorter pulses and hinder further power scaling of $\mathrm{Yb}$-doped bulk oscillators in the sub-30-fs regime.

a)

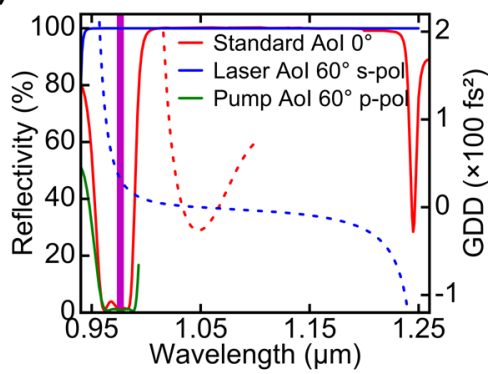

b)

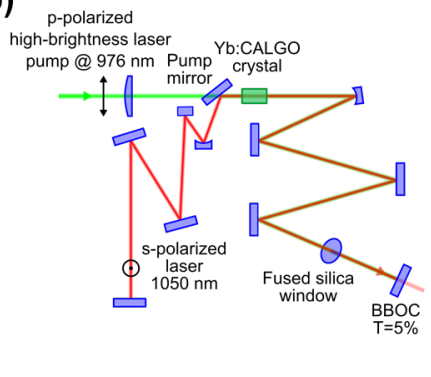

c)

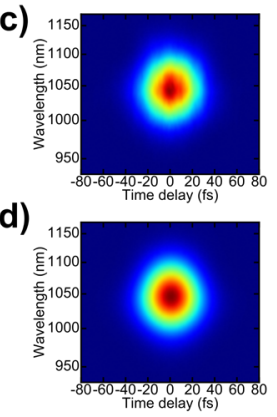

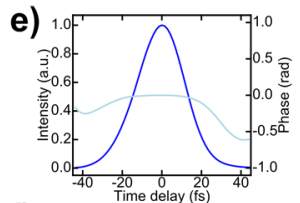

f)

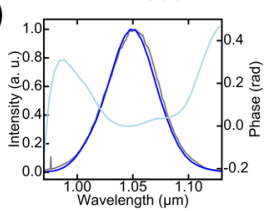

Fig. 1 a) Typical reflectivity (solid line) and GDD (dashed line) of a standard dichroic mirror (red) in comparison to the developed novel pump mirror (blue), pump wavelength at $976 \mathrm{~nm}$ (violet line). b) Schematic of the laser oscillator. c-d) Measured and retrieved SHG-FROG trace. e) Retrieved pulse intensity profile. f) Optical spectrum of the generated pulses and retrieved spectral phase.

Here, we show that we can overcome these spectral and power limitations by using a novel pump mirror design relying on cross-polarized optical pumping. The optical coatings utilized in our experimental setup [Fig. 1b)] have been designed and manufactured by ion-beam-sputtering in our facility. The developed pump mirror is highly transmissive for the pump light in p-polarization, while it is highly reflective for the laser light in s-polarization, even covering the pump wavelength and beyond [Fig. 1a)]. In contrast to a standard thin-film polarizer, it provides a low and flat GDD over a broad spectral range for the laser polarization. Applying this pumping scheme to a softaperture Kerr-lens mode-locked Yb:CALGO bulk laser oscillator, we achieve $0.5 \mathrm{~W}$ of average output power in 28 -fs pulses with an optical-to-optical efficiency of 15\%. The optical spectrum has a FWHM bandwidth of $55 \mathrm{~nm}$ that already starts to extend even beyond the pump wavelength. A SHG-FROG measurement confirms the generation of almost ideal transform-limited soliton pulses [Fig. 1c-f)]. By increasing the OC rate from 5\% to $18 \%$, we achieved $1.5 \mathrm{~W}$ of average power with slightly longer 31-fs pulses at an optical-to-optical efficiency of $33 \%$.

We overcome the power and efficiency limitations of colinearly-pumped Yb-doped bulk oscillators utilizing a novel cross-polarized optical pumping scheme. Applied to an Yb:CALGO bulk oscillator, we demonstrate up to one order of magnitude higher average power and efficiency compared to other Yb-doped bulk oscillators in the same pulse duration regime [3-5]. As we did not encounter any thermal issues in the gain medium, we expect this approach to enable efficient Yb-doped bulk oscillators operating at the multi-Watt-level in the sub-30-fs regime delivering clean soliton pulses.

\section{References}

[1] U. Morgner, F. X. Kärtner, S. H. Cho, Y. Chen, H. A. Haus, J. G. Fujimoto, E. P. Ippen, V. Scheuer, G. Angelow, et T. Tschudi, "Subtwo-cycle pulses from a Kerr-lens mode-locked Ti:sapphire laser," Optics Letters 24(6), 411 (1999).

[2] D. H. Sutter, G. Steinmeyer, L. Gallmann, N. Matuschek, F. Morier-Genoud, U. Keller, V. Scheuer, G. Angelow, et T. Tschudi,

"Semiconductor saturable-absorber mirror-assisted Kerr-lens mode-locked Ti:sapphire laser producing pulses in the two-cycle regime," Optics Letters 24(9), 631 (1999).

[3] J. Ma, X. Xu, D. Shen, et D. Tang, "21-fs Kerr-lens Mode-locked Yb:CaYAlO4 Laser," in Conference on Lasers and Electro-Optics (2018) (Optical Society of America, 2018), paper JTh2A.158.

[4] S. Kimura, S. Tani, et Y. Kobayashi, "Raman-assisted broadband mode-locked laser," Scientific Reports 9(1)(2019).

[5] P. Sévillano, P. Georges, F. Druon, D. Descamps, et E. Cormier, "32-fs Kerr-lens mode-locked Yb:CaGdAlO 4 oscillator optically pumped by a bright fiber laser," Optics Letters 39(20), 6001-6004 (2014). 